\title{
Increased Neutrophil Extracellular Traps Related to Smoking Intensity and Subclinical Atherosclerosis in Patients with Type 2 Diabetes
}

\author{
Antonios Chatzigeorgiou ${ }^{1,2, *}$ Ioannis Mitroulis ${ }^{2,3, *}$ Akrivi Chrysanthopoulou ${ }^{3}$ Aigli-loanna Legaki ${ }^{1}$ \\ Konstantinos Ritis ${ }^{3}$ Nikolaos Tentolouris ${ }^{4}$ Athanase D. Protogerou ${ }^{5}$ Michael Koutsilieris ${ }^{1, * *}$ \\ Petros P. Sfikakis ${ }^{4, * *}$
}

${ }^{1}$ Department of Physiology, Medical School, National and Kapodistrian University of Athens, Athens, Greece

2 Institute for Clinical Chemistry and Laboratory Medicine, University Clinic Carl Gustav Carus, Technische Universität Dresden, Dresden, Germany

${ }^{3}$ First Department of Internal Medicine and Laboratory of Molecular Hematology, Department of Medicine, Democritus University of Thrace, Alexandroupolis, Greece

${ }^{4}$ First Department of Propaedeutic Internal Medicine, Medical School, National and Kapodistrian University of Athens, Athens, Greece

${ }^{5}$ Cardiovascular Prevention and Research Unit, Department of Pathophysiology, Medical School, National and Kapodistrian University of Athens, Athens, Greece

Thromb Haemost 2020;120:1587-1589.

Atherosclerosis is a complex chronic inflammatory process of medium to large-sized arterial vessels, which leads to cardiovascular diseases (CVDs), including coronary artery disease and cerebrovascular disease. Endothelial cell dysfunction and activation, leading to infiltration and accumulation of inflammatory leukocytes in the subendothelial space, are key steps in the generation of atherosclerotic plaques. ${ }^{1}$ Beyond endothelial cells and platelets, neutrophils have recently emerged as important players in the development of atherosclerosis, through the release of neutrophil extracellular traps (NETs), ${ }^{2}$ which are extracellular structures composed of DNA and neutrophil peptides. ${ }^{3}$ Studies in the Apoe ${ }^{-l-}$ mouse model suggest that neutrophils contribute to atherogenesis, via the release of NETs. ${ }^{4}$ However, studies in human plaque samples suggest that neutrophils and NETs are present only in unstable plaques and upon plaque rupture, ${ }^{2,5}$ being the main leukocyte population present in thrombi at the atherothrombotic sites. ${ }^{6,7}$

Increasing lines of evidence suggest bidirectional interplay between NETs and platelets in thrombotic disorders. For instance, in arterial and venous thrombosis, activated platelets drive NET formation factors either through direct or indirect

\footnotetext{
* These authors contributed equally as first authors to the work.

** These authors contributed equally as senior authors to the work.
}

received

February 27, 2020

accepted after revision

June 12,2020

\begin{abstract}
Address for correspondence Antonios Chatzigeorgiou, MD, PhD, Department of Physiology, Medical School, National and Kapodistrian University of Athens, Mikras Asias 75, Athens 11527, Greece (e-mail: achatzig@med.uoa.gr).

Ioannis Mitroulis, MD, PhD, First Department of Internal Medicine and Laboratory of Molecular Hematology, Department of Medicine, Democritus University of Thrace, Dragana, Alexandroupolis 68100, Greece (e-mail: imitroul@med.duth.gr).
\end{abstract}

interactions with neutrophils. ${ }^{6,8,9}$ Additionally, NETs can activate endothelial cells and induce endothelial damage. 10,11 Several reports suggest that hyperglycemia in diabetes mellitus results in NET formation. ${ }^{12,13}$ However, whether NETs are linked to endothelial cell damage and atherosclerosis in diabetes mellitus in the clinical setting is largely unknown.

Based on the above, we investigated in patients with diabetes mellitus (1) the association between, on one hand, intravascular levels of NET remnants, levels of endothelial cell, and platelet activation biomarkers and, on the other hand, the degree of atherosclerosis, as assessed by the presence of nonstenotic atherosclerotic plaques in a total of eight arterial sites, and (2) the association between NET remnants, endothelial cell, and platelet activation and metabolic biomarkers (-Supplementary Table S1, available in the online version). Details are described in the - Supplementary Methods (available in the online version). To this end, diabetic patients free of CVD with either minimal ( 0 or 1 arterial site with atherosclerotic plaque) or extensive (more than five arterial sites with atherosclerotic plaques) subclinical atherosclerosis were recruited and analyzed.

To perform analysis of NET remnants, as well as of platelet and endothelial cell-derived activation biomarkers, plasma derived from EDTA-anticoagulated blood was collected. Levels

(c) 2020 Georg Thieme Verlag KG Stuttgart · New York
DOI https://doi.org/ $10.1055 / \mathrm{s}-0040-1714371$. ISSN 0340-6245. 

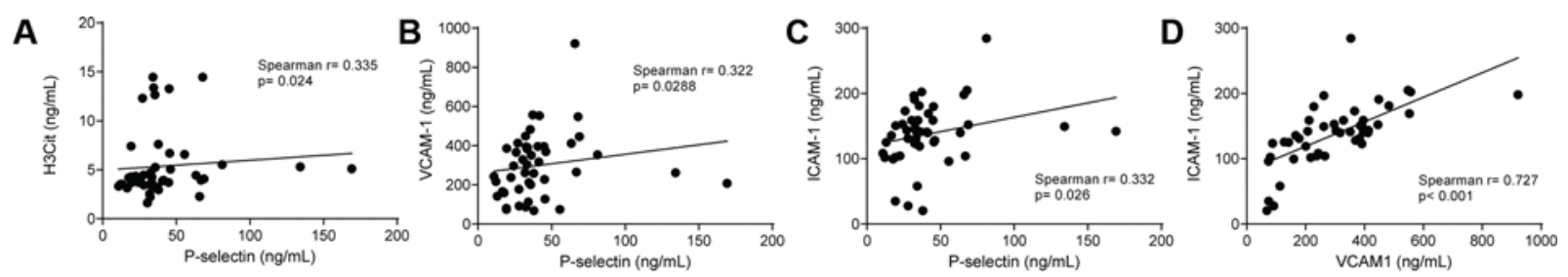

E

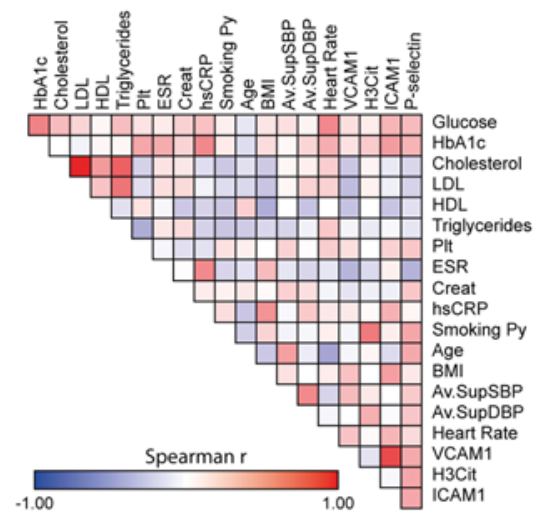

$\mathbf{F}$

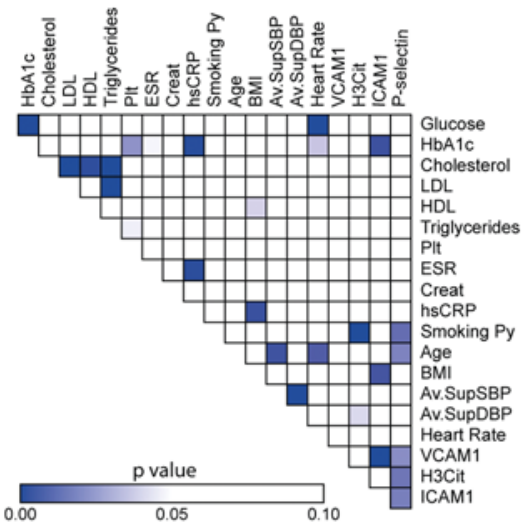

K
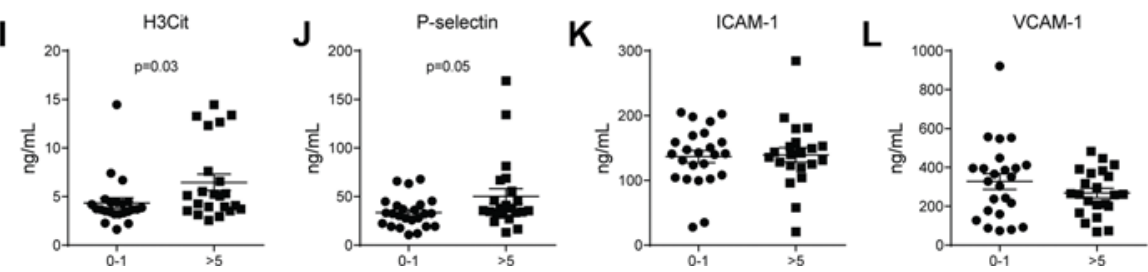

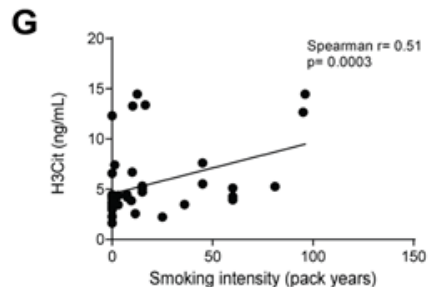

H

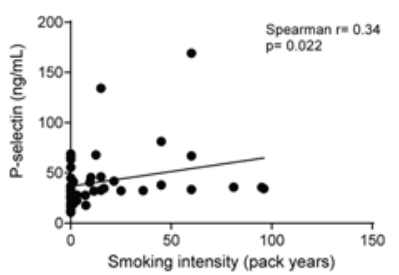

Fig. 1 (A-D) Correlation between H3Cit, ICAM-1, VCAM-1, and P-selectin in the plasma of patients with diabetes. Statistical analysis was performed using Spearman's rank correlation coefficient. (E and F) Correlation between the levels of soluble factors presented in A-D with clinical and laboratory parameters. (G and H) Correlation between H3Cit and P-selectin with smoking intensity (pack years) (I-L) Levels of H3Cit, platelet, and endothelial cell activation in patients with minimal atherosclerosis, defined as less than one atherosclerotic plaque, and patients with extensive atherosclerosis, defined as more than five atherosclerotic plaques. Statistical analysis was performed by using the MannWhitney $\mathrm{U}$ test. Data presented as mean $\pm \mathrm{SEM} . \mathrm{N}=24$ and 22, respectively. BMI, body mass index; CPK, creatine phosphokinase; ESR, erythrocyte sedimentation rate; HDL, high-density lipoprotein; hSCRP, high-sensitivity C-reactive protein; LDL, low-density lipoprotein; SEM, standard error of mean.

of NET-derived remnants were measured by engaging an enzyme-linked-immunosorbent serologic assay (ELISA) that quantifies the levels of citrullinated histone $\mathrm{H} 3$ (H3Cit), as previously described. ${ }^{14,15}$ Levels of circulating P-selectin, vascular cell adhesion protein (VCAM)-1, and intercellular adhesion molecule (ICAM)- 1 were measured by ELISA. To study whether there is a coordinated activation of neutrophils, platelets, and endothelial cells, Spearman's rank correlation analysis was performed. We observed that the levels of H3Cit were correlated with the levels of P-selectin ( - Fig. 1A), whereas P-selectin levels were also positively correlated with the levels of the endothelial cell activation markers VCAM- 1 and ICAM-1 (-Fig. 1B, C). As expected, there was a strong correlation among the levels of ICAM-1 and VCAM-1 (-Fig. 1D). However, no significant correlation was observed between the levels of $\mathrm{H} 3 \mathrm{Cit}$ and endothelial cell activation markers (-Fig. 1E, F).

We further performed correlation analysis between these markers and cardiometabolic parameters. There was a significant positive correlation between the levels of H3Cit and P-selectin with smoking intensity (measured as packyears; -Fig. 1G, H), and H3Cit levels with diastolic blood pressure; however, there was no other significant correlation with other clinical parameters, such as age, body mass index (BMI), systolic arterial pressure or heart rate, or metabolic parameters such as total cholesterol, triglycerides, low-density lipoprotein, glucose, and HbA1c levels (-Fig. 1E, F). Interestingly, there was no correlation between circulating H3Cit levels and erythrocyte sedimentation rate or high sensitivity C-reactive protein ( $\mathbf{F i g}$. 1E, F), suggesting that the levels of NET remnants do not reflect the inflammatory burden per se. Additionally, P-selectin levels were independent of the absolute platelet counts ( - Fig. 1E, F). On the other hand, the levels of the endothelial cell activation marker ICAM-1 were strongly associated with the levels of Hba1c and BMI (-Fig. 1E, F), suggesting that endothelial damage, rather than neutrophil activation, reflects diabetes control.

We further studied whether there is an association between the degree of atherosclerosis and endothelial cell, platelet, or neutrophil activation. We observed that the levels of $\mathrm{H} 3 \mathrm{Cit}$ were increased in patients with more extensive atherosclerosis (-Fig. 1I), whereas there was no statistically significant difference in the levels of P-selectin (-Fig. 1]), ICAM-1 (-Fig. 1K), or VCAM-1 (-Fig. 1L). 
In conclusion, our data indicate that NET formation is increased in diabetic patients with extended compared with minimal subclinical atherosclerosis. Our findings are in line with previous studies showing that elevated levels of circulating markers for NET formation, such as H3Cit, are associated with severe coronary arteriosclerosis, ${ }^{16}$ adverse clinical outcome in patients with stable coronary artery disease, ${ }^{17}$ myocardial infarction, ${ }^{18}$ or acute ischemic stroke. ${ }^{19}$ Interestingly, there was no association between the degree of hyperglycemia or systolic hypertension and NET formation or platelet activation in our study, factors that have been previously described as NET inducers. ${ }^{13,20}$ However, further mechanistic studies are necessary to support the pathogenic role of NETs in diabetes-associated atherosclerosis.

\section{Conflict of Interest}

None declared.

\section{References}

1 Weber C, Noels H. Atherosclerosis: current pathogenesis and therapeutic options. Nat Med 2011;17(11):1410-1422

2 Van Avondt K, Maegdefessel L, Soehnlein O. Therapeutic targeting of neutrophil extracellular traps in atherogenic inflammation. Thromb Haemost 2019;119(04):542-552

3 Boeltz S, Amini P, Anders H-J, et al. To NET or not to NET: current opinions and state of the science regarding the formation of neutrophil extracellular traps. Cell Death Differ 2019;26(03):395-408

4 Warnatsch A, Ioannou M, Wang Q Papayannopoulos V. Inflammation. Neutrophil extracellular traps license macrophages for cytokine production in atherosclerosis. Science 2015;349(6245):316-320

5 Pertiwi KR, van der Wal AC, Pabittei DR, et al. Neutrophil extracellular traps participate in all different types of thrombotic and haemorrhagic complications of coronary atherosclerosis. Thromb Haemost 2018;118(06):1078-1087

6 Stakos DA, Kambas K, Konstantinidis T, et al. Expression of functional tissue factor by neutrophil extracellular traps in culprit artery of acute myocardial infarction. Eur Heart J 2015;36(22):1405-1414

7 Naruko T, Ueda M, Haze K, et al. Neutrophil infiltration of culprit lesions in acute coronary syndromes. Circulation 2002;106(23): 2894-2900

8 Maugeri N, Campana L, Gavina M, et al. Activated platelets present high mobility group box 1 to neutrophils, inducing autophagy and promoting the extrusion of neutrophil extracellular traps. J Thromb Haemost 2014;12(12):2074-2088

9 McDonald B, Davis RP, Kim S-J, et al. Platelets and neutrophil extracellular traps collaborate to promote intravascular coagulation during sepsis in mice. Blood 2017;129(10):1357-1367

10 Folco EJ, Mawson TL, Vromman A, et al. Neutrophil extracellular traps induce endothelial cell activation and tissue factor production through interleukin- $1 \alpha$ and cathepsin G. Arterioscler Thromb Vasc Biol 2018;38(08):1901-1912

11 Gavriilaki E, Chrysanthopoulou A, Sakellari I, et al. Linking complement activation, coagulation, and neutrophils in transplantassociated thrombotic microangiopathy. Thromb Haemost 2019; 119(09):1433-1440

12 Wang L, Zhou X, Yin Y, Mai Y, Wang D, Zhang X. Hyperglycemia induces neutrophil extracellular traps formation through an nadph oxidase-dependent pathway in diabetic retinopathy. Front Immunol 2019;9:3076

13 Bryk AH, Prior SM, Plens K, et al. Predictors of neutrophil extracellular traps markers in type 2 diabetes mellitus: associations with a prothrombotic state and hypofibrinolysis. Cardiovasc Diabetol 2019;18(01):49

14 Grilz E, Mauracher L-M, Posch F, et al. Citrullinated histone H3, a biomarker for neutrophil extracellular trap formation, predicts the risk of mortality in patients with cancer. Br J Haematol 2019; 186(02):311-320

15 Mauracher L-M, Posch F, Martinod K, et al. Citrullinated histone H3, a biomarker of neutrophil extracellular trap formation, predicts the risk of venous thromboembolism in cancer patients. J Thromb Haemost 2018;16(03):508-518

16 Borissoff JI, Joosen IA, Versteylen MO, et al. Elevated levels of circulating DNA and chromatin are independently associated with severe coronary atherosclerosis and a prothrombotic state. Arterioscler Thromb Vasc Biol 2013;33(08):2032-2040

17 Langseth MS, Opstad TB, Bratseth V, et al. Markers of neutrophil extracellular traps are associated with adverse clinical outcome in stable coronary artery disease. Eur J Prev Cardiol 2018;25(07): 762-769

18 Liu J, Yang D, Wang X, et al. Neutrophil extracellular traps and dsDNA predict outcomes among patients with ST-elevation myocardial infarction. Sci Rep 2019;9(01):11599

19 Vallés J, Lago A, Santos MT, et al. Neutrophil extracellular traps are increased in patients with acute ischemic stroke: prognostic significance. Thromb Haemost 2017;117(10):1919-1929

20 Hofbauer T, Scherz T, Müller J, et al. Arterial hypertension enhances neutrophil extracellular trap formation via an angiotensin-II-dependent pathway. Atherosclerosis 2017;263:e67-e68 